\title{
The relationship between carotid disease and retinopathy in type 2 diabetes
}

Jocelyn J. Drinkwater, Fred K. Chen, Alison M. Brooks,

Brad T. Davis, Angus W. Turner, Danuta Sampson, Timothy M.E. Davis and Wendy A. Davis

\section{Background}

- Diabetic retinopathy is a leading cause of visual impairment, especially in younger people.

- Macrovascular disease has been associated with diabetic retinopathy but this relationship is not well established.

\section{Aims}

- To determine if carotid disease is associated with retinopathy in a community-based sample of people with type 2 diabetes.

\section{Methods}

- A random sample of 360 Fremantle Diabetes Study Phase II participants were invited to participate.

- Participants attended a single study visit between May 2018 and May 2019.

- A detailed history was taken, a physical assessment, including a carotid ultrasound and ophthalmic tests to assess diabetic retinopathy, was conducted and fasting blood and urine samples were collected.

- Data from the carotid ultrasound utilised in this analysis comprise presence and severity of any stenosis, type of plaque and intima-media thickness (IMT). Three IMT measurements at both of the bifurcation and the common carotid artery were obtained for each side and the average thickness was used in analysis.

- Diabetic retinopathy presence or absence (see Figure 1) was assessed from fundus photography, according to the Early Treatment Diabetic Retinopathy Study protocol by an experienced ophthalmologist.

- The generalised estimating equation extension of generalised linear modelling was used to adjust for the correlation between the two eyes of one person, enabling both eyes to be included in the analysis.

- Ipsilateral carotid disease variables were added to the most parsimonious model for diabetic retinopathy, to determine if any added significantly.

\section{Results}

- 273 participants were recruited. 524 eyes from 270 participants were included in the analysis after excluding ungradeable images and those with ipsilateral endarterectomy.
- Their mean \pm SD age was $72.1 \pm 9.2$ years, $57 \%$ were male and their median [IQR] diabetes duration was 15.5 [11.322.4] years.

- After adjustment for clinically significant variables, a mean common carotid IMT >1mm was associated with significantly higher odds of any diabetic retinopathy in the ipsilateral eye (see Table 1).

Table 1: Independent associates of any diabetic retinopathy

\begin{tabular}{lcc} 
Variable & Odds ratio (95\% Cl) & P-Value \\
\hline $\begin{array}{l}\text { Age at diabetes diagnosis (per 1 year } \\
\text { increase) }\end{array}$ & $0.93(0.90-0.96)$ & $<0.001$ \\
$\begin{array}{l}\text { HbA1c (per } 1 \% \text { or } 11 \mathrm{mmol} / \mathrm{mol} \\
\text { increase) }\end{array}$ & $1.42(1.12-1.78)$ & 0.003 \\
\hline On insulin & $2.32(1.16-4.64)$ & 0.018 \\
\hline Ln (urinary albumin:creatinine ratio) & $1.45(1.14-1.83)$ & 0.002 \\
\hline Mean common carotid IMT >1mm & $\mathbf{2 . 4 5 ( 1 . 1 1 - 5 . 4 3 )}$ & $\mathbf{0} 0.027$ \\
\hline
\end{tabular}

* An increased risk of 1 in $\ln ($ urinary albumin:creatinine ratio) corresponds to a 2.72 -fold increase in urinary albumin:creatinine ratio

\section{Conclusions}

- This study confirms the association between macrovascular and microvascular disease in type 2 diabetes.

- The common soil hypothesis ${ }^{1}$ may contribute to this association. Alternatively, microemboli, calcium/cholesterolrich plaque fragments and/or platelet aggregates dislodged from carotid plaques may embolise to the retina, contributing to retinopathy².

- Carotid disease has been associated with cardiovascular risk ${ }^{3}$ but may also be useful in identifying those at risk of retinal pathology.

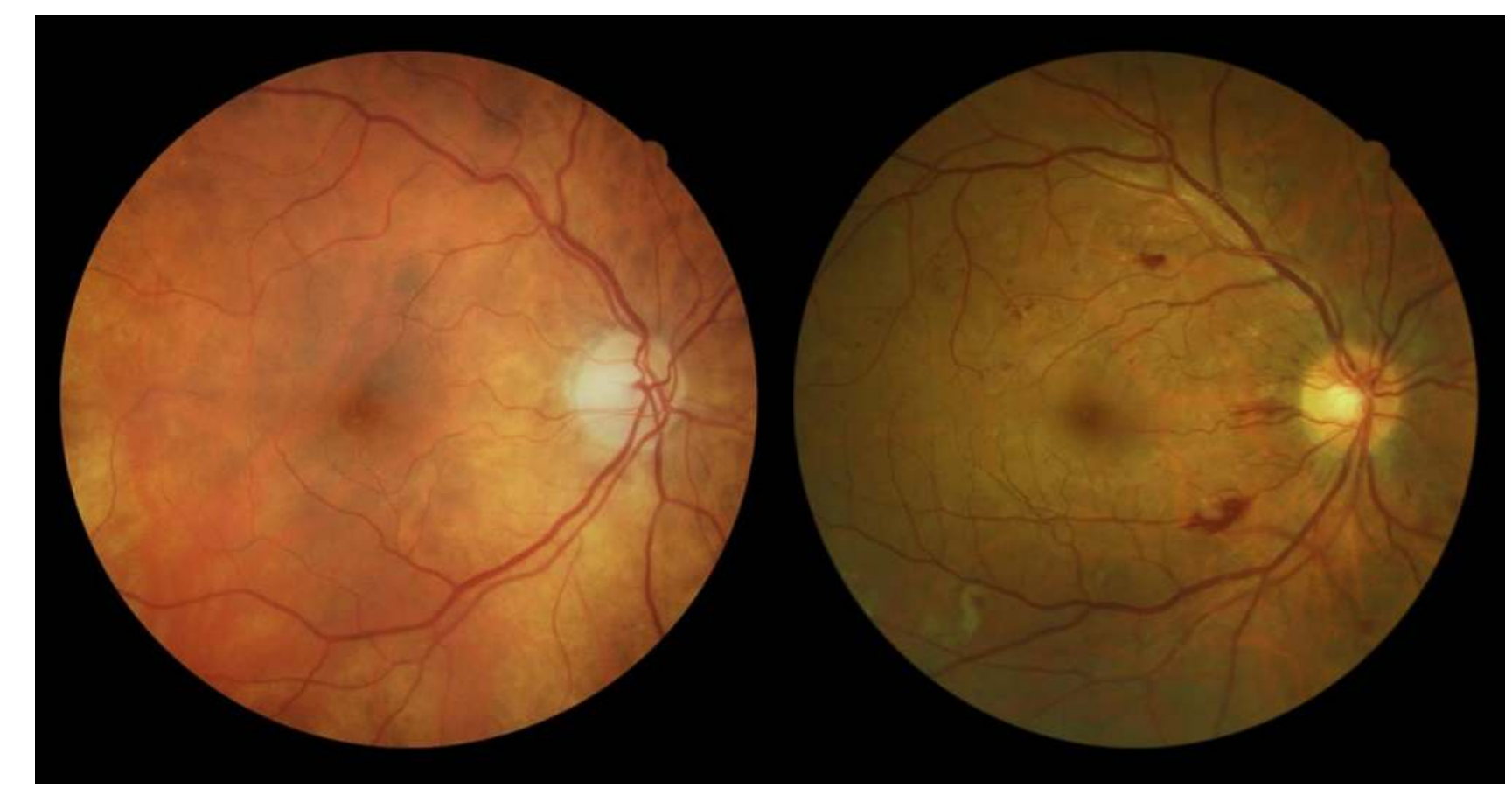

Figure 1: Images showing differences between absence (left) and presence (right) of diabetic retinopathy. 